\title{
Atenção primária à saúde para o século XXI: primeiros resultados do novo modelo de financiamento
}

\author{
Primary health care for $21^{\text {st }}$ century: first results of the new financing \\ model
}

Erno Harzheim (http://orcid.org/0000-0002-8919-7916) ${ }^{1}$

Otávio Pereira D’Avila (http://orcid.org/0000-0003-1852-7858) ${ }^{2}$

Lucas Alexandre Pedebos (http://orcid.org/0000-0001-6663-352X) ${ }^{3}$

Lucas Wollmann (http://orcid.org/0000-0002-3543-0794) ${ }^{4}$

Luis Gustavo Mello Costa (http://orcid.org/0000-0002-9678-0173) ${ }^{5}$

Carlo Roberto Hackmann da Cunha (http://orcid.org/0000-0002-3545-5801) ${ }^{6}$

Luana Nunes de Moura (http://orcid.org/0000-0002-2021-1759) ${ }^{7}$

Tales Minei (http://orcid.org/0000-0003-4380-428X) ${ }^{8}$

Livia de Almeida Faller (http://orcid.org/0000-0001-9905-0507) ${ }^{9}$
${ }^{1}$ Programa de Pós-

Graduação em

Epidemiologia, Faculdade

de Medicina, Universidade

Federal do Rio Grande do

Sul. R. Ramiro Barcelos

2.400, $2^{\circ}$ andar. 90035-003

Porto Alegre RS Brasil.

eharzheim@hcpa.edu.br

${ }^{2}$ Programa de Pós-

Graduação em Odontologia,

Faculdade de Odontologia,

Universidade Federal de

Pelotas. Porto Alegre RS

Brasil.

${ }^{3}$ Secretaria Municipal de

Saúde de Florianópolis.

Florianópolis SC Brasil.

${ }^{4}$ Grupo Hospitalar

Conceição. Porto Alegre RS

Brasil.

${ }^{5}$ Ministério das

Comunicações. Brasília DF

Brasil.

${ }^{6}$ Médico de família e

comunidade. Florianópolis

SC Brasil.

${ }^{7}$ Clínica Salute. Porto Alegre

RS Brasil.

${ }^{8}$ Médico clínico geral. Porto

Alegre RS Brasil.

${ }^{9}$ Amil Assistência Médica

Internacional. São Paulo SP

Brasil.
Abstract Since 1994, Brazil has been offering Primary Health Care (PHC) services based on the Family Health Strategy. The ESF has achieved important results. During this period, the PHC financing model did not undergo major methodological changes. In this article, the results of the new financing model for PHC approved in a tripartite manner in 2019, Previne Brasil, are laid out, which is composed of (i) weighted capitation, (ii) incentives for specific and strategic actions and, (iii) payment for performance. These first results reveal the increase of more than 50 million people with qualified and unique registration, associated with the record number of more than 52 thousand FH / eAP teams financed by the Ministry of Health, with more than 35 thousand of which (67\%) using electronic medical records. In addition, the registration of people and professionals of the Brazilian PHC, together with SISAB, is the largest set of demographic and clinical health data in the world. These advances favor the overcoming of difficulties to achieve greater access, longitudinality and coordination of care, qualifying the Brazilian PHC in search of better health outcomes.

Key words Electronic health records, Primary care, Health evaluation, Healthcare financing
Resumo O Brasil, desde 1994, oferta serviços de atenção primária à saúde (APS) a partir da Estratégia Saúde da Família (ESF). A ESF alcançou resultados importantes. Nesse período, o modelo de financiamento da APS não sofreu alterações metodológicas importantes. Neste artigo, descreve-se os resultados do novo modelo de financiamento para APS aprovado de forma tripartite em 2019, o "Previne Brasil", que é composto por (i) capitação ponderada, (ii) incentivos a ações específicas e estratégicas e (iii) pagamento por desempenho. Esses primeiros resultados revelam o incremento de mais de 50 milhões de pessoas com cadastro qualificado e único, associado ao número recorde de mais de 52 mil equipes de Saúde da Família/Equipe de Atenção Primária (EAP) financiadas pelo Ministério da Saúde, sendo mais de 35 mil (67\%) com uso de prontuário eletrônico. Além disso, o cadastro das pessoas e dos profissionais da APS brasileira junto ao Sistema de Informação em Atenção Básica (SISAB) se configura como o maior conjunto de dados demográficos e clínicos de saúde do mundo. Esses avanços favorecem a superação das dificuldades para o alcance de maior acesso, longitudinalidade e coordenação do cuidado, qualificando a APS brasileira em busca de melhores resultados em saúde.

Palavras-chave Registros eletrônicos, Atenção primária, Avaliação em saúde, Financiamento da assistência à saúde 


\section{Introdução}

O Brasil, desde 19941, oferta à população serviços de Atenção Primária à Saúde (APS) a partir da Estratégia Saúde da Família (ESF). Ao longo de seus 27 anos, a ESF alcançou resultados importantes: redução da mortalidade infantil e de mortes evitáveis em adultos ${ }^{1-3}$. Apesar dos avanços, constata-se, principalmente na última década, dificuldade de atingir bons indicadores em ações fundamentais: oferta de cuidado a condições clínicas frequentes ${ }^{4}$; imunização ${ }^{5,6}$; controle de condições crônicas ${ }^{7}$, como diabetes, hipertensão, dislipidemia e doenças respiratórias; rastreamento do câncer ${ }^{8}$; saúde mental'; diminuição de internações por condições sensíveis; e diminuição da busca por serviços de urgências por motivos não caracterizados como urgentes ${ }^{10,11}$. Soma-se a esse cenário um histórico de fragilidade em garantir acesso $^{12-17}$ e uma limitada capacidade de incorporar tecnologias, além da baixa produtividade representada pelo reduzido número de consultas por médico/ano (1.470, contra 2.294 nos países da OCDE $)^{12}$.

Nesse período, o modelo de financiamento para a APS não sofreu alterações, sendo baseado em critérios de porte populacional municipal, apresentando estrutura e oferta de serviços com pouco direcionamento para o alcance de resultados em saúde ou equidade, e sem garantia do acompanhamento real das pessoas adscritas à APS. Houve um evidente esforço para avaliar e monitorar resultados em saúde a partir do Programa Nacional de Melhoria do Acesso e da Qualidade (PMAQ), mas os resultados observados indicam que o programa auxiliou em melhorias nas instalações físicas das unidades e trouxe discussão sobre planejamento e organização dos serviços, mas não superou as dificuldades no alcance de melhores indicadores de saúde ${ }^{18}$.

O objetivo deste artigo é descrever e comentar os resultados do novo modelo de financiamento para a APS aprovado de forma tripartite em 2019, o Previne Brasil ${ }^{19,20}$. São apresentados dados relativos ao orçamento federal da APS, os efeitos imediatos da adoção da capitação ponderada, dos incentivos a ações específicas e estratégicas e do pagamento por desempenho.

\section{Orçamento da atenção primária à saúde}

No Gráfico 1, pode-se observar a evolução do orçamento federal para APS relativo às ações orçamentárias do novo financiamento no período entre 2018 e $2021^{21}$, e no Gráfico 2, a proporção da variação dos montantes repassados.
Observa-se que os recursos das ações orçamentárias 219A e 217U se mantinham acompanhando a perda de poder da moeda corrente brasileira em 2018 e 2019 e em 2020 ultrapassou essa perda inflacionária, ou seja, obtendo ganhos reais em sua composição orçamentária. De acordo com dados do Îndice de Preços ao Consumidor Amplo - IPCA ${ }^{22}$, a inflação do exercício de 2019 foi de $4,3 \%$, enquanto o crescimento dos recursos do novo financiamento foi de $8,4 \%$.

Por outro lado, no exercício orçamentário de 2021, até a presente data, o orçamento em APS perde relevância. Em que pese a execução de 2021 ainda estar em andamento, no Projeto de Lei Orçamentária Anual relativo ao exercício de 2022 os recursos não repõem a inflação do período. As expectativas quanto à projeção de inflação para o ano de 2021 giram em torno de $8,5 \%{ }^{22}$, enquanto o acréscimo orçamentário foi de 3,2\% até o presente momento. Já em relação ao exercício de 2022, a expectativa inflacionária do mercado financeiro prediz uma reposição de $4,1 \%$, enquanto a proposta de orçamento indica acréscimo de $6,1 \%{ }^{23}$.

\section{Capitação ponderada - responsabilização pelas pessoas e a ampliação do acesso}

Um dos focos do Previne Brasil é colocar as pessoas no centro do sistema. $\mathrm{O}$ novo modelo incentivou os municípios a identificarem os cidadãos atendidos por meio de cadastros melhor qualificados, dando as condições estruturais para uma maior responsabilização das equipes da ESF pelas pessoas. Isso, aliado a uma estratégia de informatização, permitiu um rápido crescimento da base de cidadãos devidamente identificados e com efetivo atendimento pelas equipes de Saúde da Família (eSF).

Em novembro de 2019, no lançamento do Previne Brasil, o dado do quadrimestre anterior (2019 Q2) demonstrava que, até agosto daquele ano, 98,2 milhões de pessoas estavam devidamente cadastradas no banco de dados da APS $(\mathrm{SISAB})^{24}$. Para verificação de crescimento, em comparação aos dois quadrimestres anteriores de 2019, já com a discussão do Previne Brasil ocorrendo em todos os estados da federação, o crescimento médio da base cadastral foi entre $6,4 \% \mathrm{e}$ $6,7 \%$ por quadrimestre. $\mathrm{O}$ último dado disponível, referente a agosto de 2021 (2021 Q2), mostra 151,8 milhões de pessoas devidamente cadastradas, sem duplicatas, representando um aumento de 55\% em relação ao momento do lançamento do novo financiamento, um crescimento quadrimestral de mais de $9 \%{ }^{24}$. 
Comparativo PLxPL Corrigido

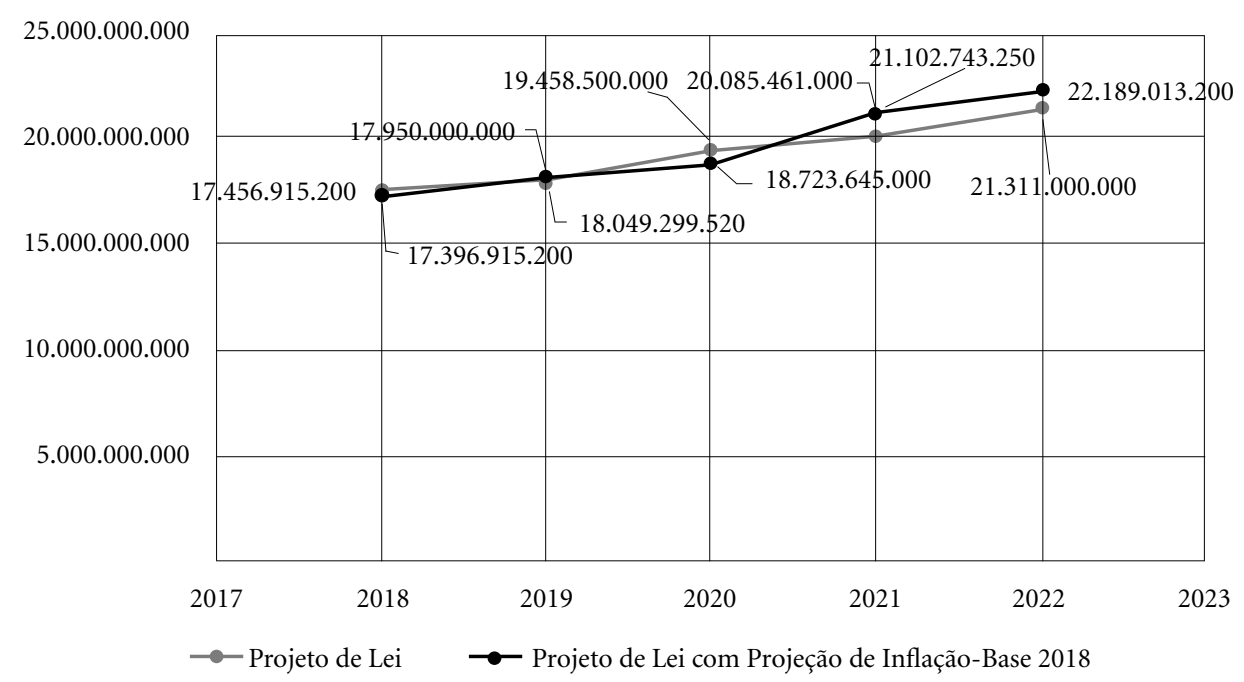

Gráfico 1. Proposta de destinação dos recursos das Ações Orçamentárias 219A e 217U (Previne Brasil), segundo Projeto de Lei e com a correção da inflação estimada, Brasil, 2021.

Fonte: Brasil, Sistema Integrado de Planejamento e Orçamento (https://www.siop.planejamento.gov.br/modulo/login/index. html $\# /)$.

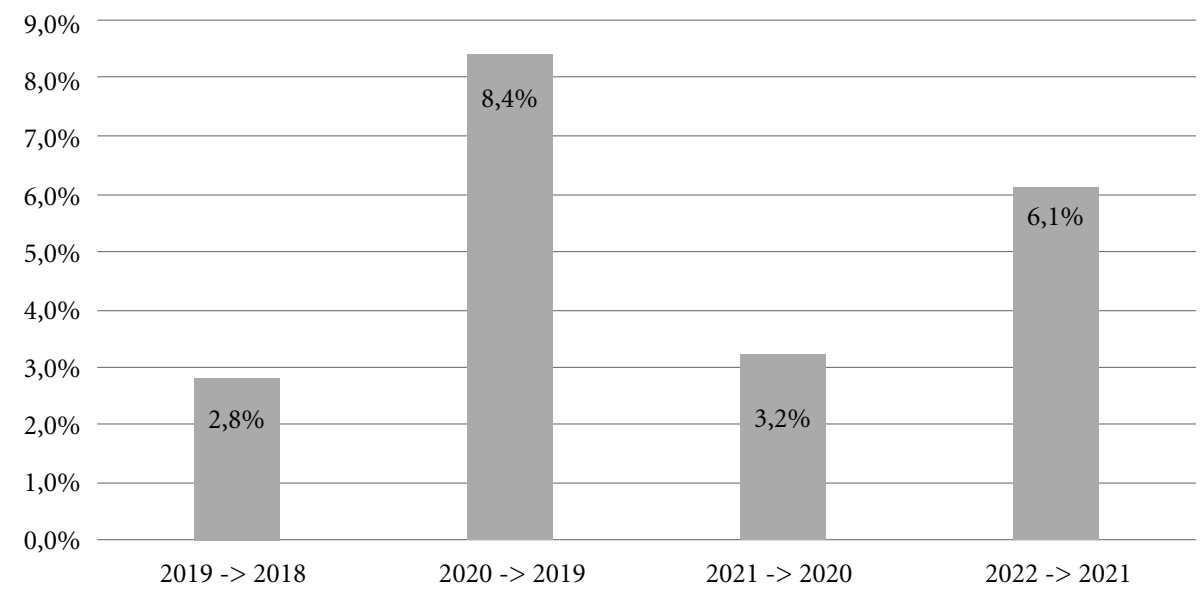

Gráfico 2. Proporção da variação orçamentária anual das ações orçamentárias 219A e 217U, Brasil, 2021.

Fonte: Brasil, Sistema Integrado de Planejamento e Orçamento (https://www.siop.planejamento.gov.br/modulo/login/index. html\#/).

O modelo de capitação também permitiu que as pessoas tivessem liberdade para se cadastrar em qualquer equipe, sem a necessidade de vinculação feita exclusivamente por critérios geográficos. Se o cidadão for cadastrado por uma eSF, mas não for efetivamente atendido, a eSF que de fato o atendeu acaba ficando com o cadastro efetivado junto ao governo federal, mesmo nos casos em que essa efetivação envolva eSF de municípios diferentes. Essa abordagem corrige distorções provocadas pelo modelo de pagamento per capita de população total (antigo PAB fixo), 
já que esse critério não destinava recursos para onde o cidadão era efetivamente atendido.

Nas últimas décadas, consolidou-se o paradigma do cuidado centrado na pessoa como elemento fundamental de qualidade em saúde. Tanto numa perspectiva estrutural dos sistemas de saúde ${ }^{25}$ como no desenvolvimento de competências profissionais de cuidado clínico ${ }^{26}$. A longitudinalidade é uma das principais estratégias para se atingir o triplo objetivo (triple aim $)^{27}$ do cuidado em saúde, uma vez que promove melhores desfechos em saúde, com maior satisfação e menor custo $^{28}$. O efeito da longitudinalidade é mediado pela relação de confiança entre as pessoas e os profissionais, estabelecida ao longo do tempo. Como é possível construir uma relação de confiança a partir de uma designação vertical de referência de cuidado baseada em território, e não na autonomia da escolha?

A orientação comunitária, atributo derivado da APS, não significa adscrição assistencial por territórios, mas sim "uma abordagem da APS que utiliza habilidades epidemiológicas e clínicas de forma complementar para ajustar os programas para que atendam às necessidades específicas de saúde de uma população definida”28. O suposto benefício do cuidado orientado para o território é a sinergia entre as ações de assistência e vigilân$\mathrm{cia}^{29}$. Contudo, no SUS há uma fragmentação entre essas duas ações. A integração entre assistência e vigilância depende da qualidade da gestão da informação clínica das pessoas, mais do que da orientação pelo território. É o fortalecimento da informatização e da integração das informações clínico-epidemiológicas da população que vai permitir às equipes trabalharem assistência e vigilância de forma conjunta, incorporando a informação contextual no cuidado individual. A necessidade da superação da adscrição orientada pelo território já vem sendo apontada por diferentes especialistas em APS no Brasil ${ }^{30} \mathrm{e}$ até pela ABRASCO $^{31}$. Se o cuidado deve ser centrado nas pessoas, oferecido por meio de relações de confiança ao longo do tempo, o vínculo necessariamente deve ser feito com as pessoas, livremente, e não com as suas casas.

No mesmo sentido, a necessidade de expansão contínua do número de serviços da APS levou à inclusão de equipes com cargas horárias flexíveis, em um modelo de trabalho pautado no desenvolvimento dos atributos da APS. O financiamento das Equipes de Atenção Primária à Saúde (eAP) com cargas horárias de $20 \mathrm{~h}$ e $30 \mathrm{~h}$ resultou num crescimento de equipes financiadas, aumentando o contingente de pessoas cadastradas e de acesso a serviços de saúde ${ }^{32}$. Em dezembro de 2018 eram 43.026 equipes de APS financiadas, um ano depois eram 43.755 , e para a competência de setembro de 2021 foram identificadas 52.829 equipes, sendo 48.611 eSF e 4.218 eAP (referência CNES de setembro de 2021) ${ }^{33}$.

\section{Informatiza APS - a valorização dos registros em saúde}

A informatização dos serviços da APS também é parte da estratégia do Previne Brasil. Em 2019, a Secretaria de Atenção Primária à Saúde (SAPS) do Ministério da Saúde lançou o Programa Informatiza APS $^{34}$ como maneira de incentivar os municípios a informatizarem suas unidades. Recursos foram repassados diretamente aos municípios, tendo como contrapartida clara e específica a qualificação dos dados enviados ao Ministério da Saúde (MS), com consequente promoção do desenvolvimento de empresas do setor por meio da livre concorrência. Com esse incentivo, os municípios passaram a escolher a solução que mais se adaptava à sua própria realidade local.

Esse elemento é intrínseco e fundamental para o Previne Brasil, já que tanto o pagamento populacional por capitação (per capita) quanto o pagamento por desempenho com base em indicadores, calculados diretamente no banco de dados federal (SISAB), necessitam de dados de qualidade coletados, processados e enviados de modo correto. Especificamente quanto ao cálculo dos indicadores para pagamento por desempenho, esses deixaram de ter base quantitativa (agregado populacional) e passaram a ser de base individualizada por cada cidadão para cálculo. Tal mudança possibilitou a adoção de diversas ferramentas de gestão da clínica, como busca-ativa, monitoramento de casos e mensuração de desfechos.

No momento da implantação do Informatiza APS, o MS adotou, de forma mais conservadora, um padrão para definir se as equipes estavam informatizadas e incluiu todas as equipes que enviaram algum dado coletado por meio de solução informatizada, seja e-SUS PEC ou solução proprietária/de terceiros. Nesse cenário, havia $27.514(62,40 \%)$ equipes com algum grau de informatização, das $44.072 \mathrm{eSF}$ em funcionamento naquele momento ${ }^{24}$. Atualmente, segundo dados do Informatiza APS, 41.117 (78,25\%) equipes possuem algum grau de informatização, estando aptas a solicitar o incentivo do programa. Ao desconsiderar equipes com envio mais irregular, 
ainda há 35.365 (69,20\%) equipes já homologadas ou deferidas ${ }^{24}$, o que representa um acréscimo de $24 \%$ em número de equipes plenamente informatizadas num período inferior a dois anos.

\section{Pagamento por desempenho}

O Previne Brasil também propôs sete indicadores de desempenho para o ano de $2020^{21,22}$. Era previsto o incremento de mais sete indicadores para 2021 e outros sete para 2022, incluindo indicadores globais de qualidade da APS, como net promoter score, os escores do PCATool-Bra$\mathrm{sil}^{35}$ e os escores do PDRQ9 ${ }^{36}$. No entanto, devido à pandemia de covid-19, o pagamento por desempenho não tem considerado o resultado real obtido. Essa situação atrasou o avanço da APS no Brasil, acumulando para os próximos anos a necessidade de mensurar e pagar de acordo com o resultado uma ampla agenda de cuidados, principalmente para condições crônicas em saúde e saúde materno-infantil. Contudo, é possível, a partir dos resultados iniciais (Tabela 1), observar um impacto positivo do uso de indicadores de desempenho para monitoramento da APS.

Infelizmente, o indicador "cobertura vacinal de poliomielite inativada e de pentavalente" não possui dados públicos atualizados para o período observado. É importante destacar que a piora ou melhora desses indicadores pode ser facilmente identificada a partir da disponibilização da informação de forma fácil e simples por parte da SAPS, que permite a avaliação individualizada do indicador até o nível das eSF. Esse feedback quadrimestral da qualidade do cuidado vai permitir que as equipes consigam melhorar o atendimento oferecido, com base em informações do seu próprio trabalho, além de garantir transparência junto à população, dois avanços importantes em relação ao PMAQ.

\section{Incentivo a ações específicas e estratégicas do Previne Brasil}

\section{Saúde na hora}

O programa Saúde na Hora, lançado em 2019, objetivava financiar equipes com horário de funcionamento estendido ${ }^{37}$. Atualmente, existem 2.600 Unidades Básicas de Saúde atuando com horário estendido, distribuídas em 595 municípios brasileiros, envolvendo 8.238 eSF, 217 eAP e 2.397 Equipes de Saúde Bucal (ESB) ${ }^{33}$.

\section{Saúde bucal}

Entre as ações do Previne Brasil que fortaleceram a saúde bucal na APS, pode-se citar a expansão das equipes de saúde bucal, a priorização de públicos estratégicos para o alcance do acesso universal à saúde bucal na APS (por exemplo: gestantes) e qualificação do cuidado, além da própria expansão do montante de recursos que financia tal fim. Para a indução da ampliação de equipes de saúde bucal, o Previne Brasil previu, para 2020, o ajuste do recurso de custeio federal das equipes de saúde bucal, ampliando-o em $10 \%{ }^{38}$, que somados ao pagamento de desempenho resultaram num aumento de custeio de $32 \%$ paras as equipes de saúde bucal ${ }^{21,39}$. Além dessa ação, foram reconhecidas também as equipes de saúde bucal que funcionam com carga horária mensal diferenciada. A medida buscou reconhecer as equipes que já estavam em funcionamento mas não usufruíam dos recursos federais por atuarem em 30 ou 20 horas semanais ${ }^{40}$. Atualmente, são 30.117 equipes de saúde bucal com carga horária de 40 horas semanais e 1.060 com 20/30 horas semanais homologadas e financiadas pelo $\mathrm{MS}^{24}$.

Tabela 1. Comparação dos resultados obtidos pelos indicadores de desempenho no período de 2018 a 2021.

\begin{tabular}{lccc}
\hline \multicolumn{1}{c}{ Indicadores de desempenho } & Meta & $\begin{array}{c}\text { Quadrimestre } \\
\text { 1 de 2018 }\end{array}$ & $\begin{array}{c}\text { Quadrimestre } \\
\text { 2 de 2021 }\end{array}$ \\
\hline $\begin{array}{l}\text { Proporção de gestantes com pelo menos seis consultas pré-natal } \\
\text { realizadas, sendo a primeira até a 20a semana de gestação. }\end{array}$ & $60 \%$ & $16 \%$ & $40 \%$ \\
Proporção de gestantes com realização de exames para sífilis e HIV. & $60 \%$ & $17 \%$ & $50 \%$ \\
Proporção de gestantes com atendimento odontológico. & $60 \%$ & $13 \%$ & $34 \%$ \\
Cobertura de exame citopatológico. & $40 \%$ & $10 \%$ & $14 \%$ \\
$\begin{array}{l}\text { Percentual de diabéticos com solicitação de hemoglobina glicada. } \\
\text { Percentual de pessoas hipertensas com pressão arterial aferida em }\end{array}$ & $50 \%$ & $7 \%$ & $17 \%$ \\
cada semestre. & & $2 \%$ & $8 \%$
\end{tabular}

Fonte: Brasil, Ministério da Saúde. Informação e Gestão da Atenção Básica (https://egestorab.saude.gov.br/). 


\section{Formar equipes com base em necessidades em saúde locais}

Outro aspecto relevante foi o fim do financiamento específico para os Núcleos de Apoio à Saúde da Família (NASF). Em setembro de 2019, havia 96.525 profissionais de saúde de nível superior passíveis de composição das equipes NASF atuando na $\mathrm{APS}^{40}$. Desse total, apenas 35\% estavam vinculados ao financiamento específico dos NASF. Com a flexibilização do financiamento, permitindo ao município estabelecer a equipe multiprofissional mais adequada de acordo com as necessidades em saúde locais, observou-se um aumento da presença desses profissionais na APS. Segundo dados do CNES de setembro de $2021^{33}$, atualmente são 111.813 profissionais de saúde de nível superior do mesmo tipo citado anteriormente atuando na APS, um incremento de $16 \%$ desde a implementação do Previne Brasil.

\section{Incentivo à formação qualificada}

O incentivo de formação profissional no âmbito da APS tem como objetivo qualificar o cuidado às pessoas com o apoio à formação profissional especializada para a atenção primária, por meio de repasse financeiro aos municípios que tenham profissionais de medicina, enfermagem ou odontologia realizando residência profissional em equipes de Saúde da Família ou equipes de saúde bucal ${ }^{41}$. O incentivo já está sendo repassado para 2.151 vagas em programas de residência, sendo 1.331 vagas em medicina, $571 \mathrm{em}$ enfermagem e 249 em odontologia, distribuídas por 120 municípios. Isso corresponde a um repasse anual de $\mathrm{R} \$ 86.634 .000,00$ aos municípios habilitado ${ }^{21}$ no apoio à consolidação de residência em APS

\section{Considerações finais}

A descrição dos primeiros resultados do Previne Brasil torna evidente o benefício à população produzido pela reforma do financiamento da APS. O incremento de mais de 50 milhões de pessoas com cadastro qualificado e único, associado ao número recorde de mais de 52 mil equipes de SF/AP financiadas pelo MS, sendo mais de $35 \mathrm{mil}$ dessas com uso constante de prontuário eletrônico, favorece sobremaneira a superação das dificuldades para o alcance de maior presença do acesso de primeiro contato, da longitudinalidade e da coordenação do cuidado, muito dependentes da continuidade da informação clínica. Além disso, o cadastro das pessoas e dos profissionais da APS brasileira junto ao SISAB se configura como o maior conjunto de dados demográficos e clínicos de saúde do mundo. Se mantidas as ações iniciadas em 2019/2020 de qualificação do e-SUS-PEC, com inclusão de variáveis clínicas fidedignas e mensuráveis, além do controle de duplicatas dos cadastros e da incorporação de informações de contato como telefones celulares e endereço por meio do cruzamento de bases de dados federais, a possibilidade de realizar grandes intervenções por meio de tecnologia de informação não terá precedentes. Será possível ter lista de pacientes/pessoas por equipe ou unidade de saúde, produzir relatórios de busca ativa - como já realizado na vacinação do sarampo em fins de 2019, monitorar pacientes com doenças crônicas, engajar pessoas com doenças crônicas em ações que aumentem adesão às terapêuticas instituídas, entre outras ações clínicas muito relevantes frente ao cenário epidemiológico de tripla carga de doenças.

Além dos benefícios percebidos pelo componente de capitação, o componente dos incentivos produziu um aumento considerável no número de unidades e equipes que funcionam até as $20 \mathrm{~h}$ ou 22h, ampliando o acesso das pessoas à APS. Houve também forte expansão das equipes de saúde bucal, da presença de profissionais de saúde e de vagas de residência para médicos, enfermeiros e odontólogos.

Esses benefícios, aliados à definição da Carteira de Serviços da $\mathrm{APS}^{42}$ e do projeto em andamento da definição de linhas de cuidado, abrem grandes possibilidade de qualificação da prática clínica na APS, criando as condições estruturais para enfrentar os desafios citados no início do texto, principalmente a qualificação do cuidado das condições crônicas, do envelhecimento e da saúde mental.

Cabe ressaltar que a indução do aumento de pessoas sob real responsabilidade da APS, do número de equipes de SF/AP, do uso de prontuário eletrônico, de equipes de saúde bucal, da composição multiprofissional das Unidades de Saúde e de vagas na residência ocorreram em 20 meses, dos quais 18 estavam sob a pressão e o desafio do enfrentamento da pandemia de COVID-19. As mudanças e conquistas descritas foram fruto muito mais da mudança de método de financiamento do que do aumento, também expressivo, no valor dos repasses federais no período. E, sem dúvida nenhuma, tem como protagonistas os gestores municipais de saúde dos 5.570 municípios brasileiros, que com apoio do CONASEMS 
e CONASS participaram ativamente da construção do Previne Brasil e de sua implantação exitosa. Mesmo à sombra da pandemia, o uso de métodos de gestão embasados em evidências e apoiados em experiências de sucesso em contexto internacional ${ }^{43}$ trouxe nova luz à APS brasilei- ra, representando inovação para o SUS. Essa luz pode ser uma esperança de que a gestão tripartite, ao privilegiar métodos e técnicas cientificamente embasados, pode trazer ao SUS o aumento do acesso e da qualidade, com sustentabilidade financeira, que a população brasileira necessita.

\section{Colaboradores}

Otávio D’Avila e Erno Harzheim contribuíram na concepção, execução, desenvolvimento e revisão final do estudo. Lucas Alexandre Pedebos, Lucas Wollmann e Luis Gustavo Mello Costa foram responsáveis pela execução, descrição e revisão dos resultados obtidos. Carlo Roberto Hackmann da Cunha, Livia de Almeida Faller, Luana Nunes de Moura e Tales Minei contribuíram com a revisão final do artigo, ajustes textuais e aprimoramento da discussão dos dados. 


\section{Referências}

1. Kringos DS, Boerma W, Van der Zee J, Groenewegen P. Europe's strong primary care systems are linked to better population health but also to higher health spending. Health Affairs 2013; 32(4):686-694.

2. Rocha R, Soares R. Evaluating the impact of community-based health interventions: evidence from Brazil's Family Health Program. Health Econnomics 2010; 129(51):126-158.

3. Bastos ML, Menzies D, Hone T, Dehghani K, Trajman A. Correction: the impact of the Brazilian family health on selected primary care sensitive conditions: a systematic review. PLoS One 2017; 12(12):e0189557.

4. Chueiri PS, Gonçalves MR, Hauser L, Wollmann L, Mengue SS, Roman R, Rodrigues Agostinho Rech M, Soares MAV, Pertile J, Harzheim E. Reasons for encounter in primary health care in Brazil. Fam Pract 2020; 37(5):648-654.

5. Arroyo LH, Ramos ACV, Yamamura M, Crispim JA, Cartagena-Ramos D, Fuentealba-Torres M, Santos DT, Palha PF, Arcêncio RA. Áreas com queda da cobertura vacinal para BCG, poliomielite e tríplice viral no Brasil (2006-2016): mapas da heterogeneidade regional. Cad Saude Publica 2020; 36(4):e00015619.

6. Domingues CMAS, Maranhão AGK, Teixeira AM, Fantinato FFS, Domingues RAS. 46 anos do Programa Nacional de Imunizações: uma história repleta de conquistas e desafios a serem superados. Cad Saude Publica 2020; 36(Suppl. 2):e00222919.

7. Reis RCP, Duncan BB, Szwarcwald CL, Malta DC, Schmidt MI. Control of glucose, blood pressure, and cholesterol among adults with diabetes: the Brazilian National Health Survey. J Clin Med 2021; 10(15):3428.

8. Chueiri PS, Gonçalves MR, Hauser L, Mengue S, Agostinho M, Roman R, Wollmann L, Dilda A, Silva RAM, Harzheim E. Brazilian Survey on Preventive Actions for the Population with Access to Primary Healthcare: inefficient spending in a country in economic crisis. Int J Health Policy Manag 2021. doi: 10.34172/ijhpm.2021.94. Epub ahead of print.

9. Salum GA, Leite LDS, Santos SJE, Mazzini G, Baeza FLC, Spanemberg L, Evans-Lacko S, Sato JR, Nascimento DM, Frank T, Pfeil J, Katz N, Osório J, Santos PR, Silva E, Nunes C, Soares KN, Machado AMG, Breyer T, Rodrigues M, Galão A, Motta GL, Schuch S, Osório E, Rodrigues C, Sturmer PL, Harzheim E. Prevalence and trends of mental disorders requiring inpatient care in the city of Porto Alegre: a citywide study including all inpatient admissions due to mental disorders in the public system from 2013-2017. Trends Psychiatry Psychother 2020; 42(1):86-91.

10. Magalhães FJ, Lima FET, Barbosa LP, Guimarães FJ, Felipe GF, Rolim KMC, Lima EPM. Risk classification of children and adolescents: priority of care in the emergency unit. Rev Bras Enferm 2020; 73(Suppl. 4):e20190679.

11. Mendes EV. As redes de atenção à saúde. Brasília: OPAS; 2011.

12. Banco Mundial. Um ajuste justo: Análise da eficiência e equidade do gasto público no Brasil. Brasília: Banco Mundial; 2017.
13. Mesquita Filho M, Luz BSR, Araújo CS. A Atenção Primária à Saúde e seus atributos: a situação das crianças menores de dois anos segundo suas cuidadoras. Cien Saude Colet 2014; 19(7):2033-2046.

14. Carvalho VCHS, Rossato SL, Fuchs FD, Harzheim E, Fuchs SC. Assessment of primary health care received by the elderly and health related quality of life: a cross-sectional study. BMC Public Health 2013; 13:605.

15. Ibañez N, Rocha JSY, Castro PC, Ribeiro MCSA, Forster AC, Novaes MHD, Viana ALA. Avaliação do desempenho da atenção básica no Estado de São Paulo. Cien Saude Colet 2006; 11(3):683-703.

16. Harzheim E, Hauser L, Pinto LF. Avaliação do grau de orientação para Atenção Primária em Saúde: a experiência dos usuários das Clínicas da Família e Centros Municipais de Saúde na cidade do Rio de Janeiro (relatório final da pesquisa PCATool - Rio-2014). Porto Alegre: UFRGS; 2015.

17. Pinto LF, Harzheim E, Hauser L, D'Avila OP, Gonçalves MR, Travassos P, Pessanha R. A qualidade da Atenção Primária à Saúde na Rocinha - Rio de Janeiro, Brasil, na perspectiva dos cuidadores de crianças e dos usuários adultos. Cien Saude Colet 2017; 22(3):771781.

18. Sellera PEG, Pedebos LA, Harzheim E, Medeiros OL, Ramos LG, Martins C, D'ávila OP. Monitoramento e avaliação dos atributos da Atenção Primária à Saúde em nível nacional: novos desafios. Cien Saude Colet 2020; 25(4):1401-1412.

19. Brasil. Portaria no 2.979, de 12 de novembro de 2019. Institui o Programa Previne Brasil, que estabelece novo modelo de financiamento de custeio da Atenção Primária à Saúde no âmbito do Sistema Único de Saúde, por meio da alteração da Portaria de Consolidação no 6/GM/MS, de 28 de setembro de 2017.

20. Harzheim E, D'ávila OP, Ribeiro DC, Ramos LG, Silva LE, Santos CMJ, Costa LGM, Cunha CRH, Pedebos LA. Novo financiamento para uma nova Atenção Primária à Saúde no Brasil. Cien Saude Colet 2020; 25(4):1361-1374.

21. Brasil. SIOP Consulta Livre. [acessado 2021 Out 18]. Disponível em: https://wwwl.siop.planejamento.gov. br/QvAJAXZfc/opendoc.htm?document=IAS\%2F Execucao_Orcamentaria.qvw\&host $=$ QVS\% 40 pqlk 04\&anonymous $=$ true\&sheet $=$ SH06

22. Instituto Brasileiro de Geografia e Estatística. IPCA - Índice Nacional de Preços ao Consumidor Amplo. [acessado 2021 Out 18]. Disponível em: https://www. ibge.gov.br/estatisticas/economicas/precos-e-custos/ 9256-indice-nacional-de-precos-ao-consumidor-amplo.html? $=\& \mathrm{t}=$ series-historicas

23. Banco Central do Brasil. Focus - Relatório de Mercado de 27/09/2021. [acessado 2021 Out 18]. Disponível em: https://www.bcb.gov.br/content/focus/focus/ R20210924.pdf

24. Brasil. Sistema de Informação em Saúde para a Atenção Básica. [acessado 2021 Out 18]. Disponível em: https://sisab.saude.gov.br/paginas/acessoRestrito/relatorio/federal/saude/RelSauProducao.xhtml 
25. Committee on Quality Health Care in America, Institute of Medicine. Crossing the Quality Chasm: a New Health System for the 21st Century. Washington, D.C.: National Academy Press; 2001.

26. Stewart M, Welston W, Mcwillian C. Medicina centrada na pessoa: transformando o método clínico. Porto Alegre: Artmed, 2017.

27. Berwick DM, Nolan TW, Whittington J. The triple aim: care, health, and cost. Health Aff (Millwood) 2008; 27(3):759-69.

28. Starfield Bárbara. Atenção primária: equilíbrio entre necessidade de saúde, serviços e tecnologia. Brasília: UNESCO, Ministério da Saúde; 2004.

29. Teixeira CF, Paim JS, Vilasbôas AL. SUS, modelos assistenciais e vigilância da saúde. Inf Epidemiol 1998; 7(2):7-28.

30. Tasca R, Massuda A, Carvalho WM, Buchweitz C, Harzheim E. Recomendações para o fortalecimento da atenção primária à saúde no Brasil. Rev Panam Salud Publica 2020; 44:e4.

31. Associação Brasileira de Saúde Coletiva (ABRASCO) Fortalecer o SUS, em defesa da democracia e da vida [Internet]. 2020. [acesso 2021 Out 3]. Disponível em: https://www.abrasco.org.br/site/wp-content/uploads/2020/10/Abrasco_Fortalecer-o-SUS.pdf

32. Brasil. Portaria no 2.539, de 26 de setembro de 2019. Altera as Portarias de Consolidação no 2/GM/MS, de 28 de setembro de 2017 , e n ${ }^{\circ} 6$, de 28 de setembro de 2017, para instituir a equipe de Atenção Primária eAP e dispor sobre o financiamento de equipe de Saúde Bucal - eSB com carga horária diferenciada.

33. Brasil. Cadastro Nacional de Estabelecimentos em Saúde. [acesso 3 Ago 2020]. Disponível em: http:// cnes.datasus.gov.br/

34. Brasil. Portaria no 2.983, de 11 de novembro de 2019. Institui o Programa de Apoio à Informatização e Qualificação dos Dados da Atenção Primária à Saúde - Informatiza APS, por meio da alteração das Portarias de Consolidação no 5/GM/MS e no 6/GM/MS, de 28 de setembro de 2017.

35. Brasil. Ministério da Saúde (MS). Secretaria de Atenção Primária à Saúde. Departamento de Saúde da Família. Manual do Instrumento de Avaliação da Atenção Primária à Saúde: PCATool-Brasil-2020. Brasília: MS; 2020.

36. Wollmann L, Hauser L, Mengue SS, Agostinho MR, Roman R, Feltz-Cornelis CMV, Harzheim E. Cross-cultural adaptation of the Patient-Doctor Relationship Questionnaire (PDRQ-9) in Brazil. Rev Saude Publica 2018; 52:71.

37. Brasil. Portaria no 930, de 15 de maio de 2019. Institui o Programa "Saúde na Hora", que dispõe sobre o horário estendido de funcionamento das Unidades de Saúde da Família, altera a Portaria nº 2.436/GM/MS, de 2017, a Portaria de Consolidação no 2/GM/MS, de 2017, a Portaria de Consolidação no 6/GM/MS, de 2017, e dá outras providências.
38. Brasil. Portaria $\mathrm{n}^{\circ} 2.305$, de 28 de agosto de 2020. Altera a Portaria de Consolidação no 6/GM/MS, de 28 de setembro de 2017, para reajustar os valores dos incentivos financeiros de custeio das Equipes de Saúde Bucal, nas modalidades 1 e 2, segundo critérios estabelecidos pela Política Nacional de Atenção Básica.

39. Brasil. Lei no 13.978, de 17 de janeiro de 2020 (http:// www.planalto.gov.br/ccivil_03/_ato2019-2022/2020/ lei/l13978.htm).

40. Brasil. Portaria no 2.918, de 21 de outubro de 2020 . Credencia municípios a receberem incentivos financeiros referentes às Equipes de Saúde Bucal com carga horária diferenciada.

41. Brasil. Portaria no 3.510, de 18 de dezembro de 2019. Altera a Portaria de Consolidação nº 6/GM/MS, de 28 de setembro de 2017, para instituir incentivo financeiro de custeio adicional mensal para municípios com equipes de saúde integradas a programas de formação profissional no âmbito da Atenção Primária à Saúde.

42. Brasil. Ministério da Saúde (MS). Secretaria de Atenção Primária à Saúde. Departamento de Saúde da Família. Carteira de serviços da Atenção Primária à Saúde (CaSAPS): versão profissionais de saúde e gestores. Brasília: MS; 2020.

43. Chasing C, Chi Y-L, Smith P, Borowitz M, Thomson S. Paying for performance in health care - implications for health system performance and accountability. Genebra: European Observatory on Health Systems and Policies; 2014

Artigo apresentado em 30/09/2021

Aprovado em 20/10/2021

Versão final apresentada em 22/10/2021

Editores-chefes: Maria Cecília de Souza Minayo, Romeu Gomes, Antônio Augusto Moura da Silva 
